\title{
ESTIMATIVA DO PLASTOCRONO EM CULTIVARES DE SOJA $\left({ }^{1}\right)$
}

\author{
NEREU AUGUSTO STRECK $\left({ }^{2 *}\right)$; GIZELLI MOIANO DE PAULA $\left({ }^{3}\right)$; CÁTIA CAMERA $\left({ }^{3}\right)$; \\ NILSON LEMOS DE MENEZES $\left({ }^{2}\right)$; ISABEL LAGO $\left({ }^{4}\right)$
}

\begin{abstract}
RESUMO
Um parâmetro bastante usado em modelos de simulação do desenvolvimento de espécies dicotiledôneas é o intervalo de tempo entre o aparecimento de dois nós sucessivos em uma haste, conhecido como plastocrono, com unidade de ${ }^{\circ} \mathrm{C}$ dia nó ${ }^{-1}$. O objetivo deste trabalho foi estimar o plastocrono em algumas cultivares de soja (Glycine $\max ($ L.) Merrill), recomendadas para cultivo no Sul do Brasil. Foram desenvolvidos dois experimentos em Santa Maria (RS), um no ano agrícola de 2004/2005 e outro em 2005/2006. Seis cultivares (BRS 66, CD 201, CD 203, CD 205, FT 9, FUNDACEP 39) foram avaliadas em 2004/2005 e cinco (CD 205, CD 209, CD 213 RR, CD 214 RR, CD 219 RR) em 2005/2006, no delineamento de blocos ao acaso, com duas repetições (média de cinco plantas) em $2004 / 2005$ e três repetições (média de seis plantas), em 2005/2006. O plastocrono foi estimado pelo inverso do coeficiente angular da regressão linear do número de nós visíveis na haste principal com a soma térmica (temperatura base de $10^{\circ} \mathrm{C}$ e temperatura ótima de $30^{\circ} \mathrm{C}$ ) diária acumulada a partir da emergência. Em muitas cultivares (CD 201, FT 9 e CD 205 em 2004/2005 e CD 219 RR, CD 205 e CD 214 RR em 2005/2006) observaram-se valores similares de plastocrono. Considerando todas as cultivares, o plastocrono médio e a variação (menor e maior valor) em 2004/2005 (ano seco) foi de 55,9 $(49,6-60,3){ }^{\circ} \mathrm{C}$ dia nó ${ }^{-1}$ e em 2005/2006 (ano úmido) foi de 50,7 $(48,1-53,9){ }^{\circ} \mathrm{C}$ dia nó ${ }^{-1}$ respectivamente. Esses resultados associados ao fato do plastocrono da cultivar CD 205, usada nos dois anos, também ter sido maior no ano mais seco, indica efeito do ambiente (déficit hídrico no solo) sobre o plastocrono.
\end{abstract}

Palavras-chave: aparecimento de nós, temperatura, desenvolvimento vegetal, graus-dia, défcit hídrico.

\section{ABSTRACT ESTIMATING THE PLASTOCHRON IN SOYBEAN CULTIVARS}

A parameter widely used in development simulation models of dicot species is the time interval between the appearance of two successive nodes on a stem, known as plastochron, with units of ${ }^{\circ} \mathrm{C}$ day node ${ }^{-1}$. The objective of this study was to estimate the plastochron in some soybean (Glycine max (L.) Merrill) cultivars recommended for Southern Brazil. Two experiments were carried out at Santa Maria, RS, Brazil, one during the 2004/2005 and another during the 2005/2006 growing season. Six cultivars (BRS 66, CD 201, CD 203, CD 205, FT 9, FUNDACEP 39) were used in 2004/2005 and five cultivars (CD 205, CD 209, CD 213 RR, CD 214 RR, CD 219 RR) in 2005/2006, in a complete randomized block design, with two replications (average of five plants) in 2004/2005 and three replications (average of six plants) in 2005/2006. The plastochron was estimated by the inverse of the slope of the linear regression of the number of visible nodes on the main stem against the accumulated daily thermal time (base temperature of $10^{\circ} \mathrm{C}$ and an optimum threshold temperature of $30^{\circ} \mathrm{C}$ ) from emergence. Many cultivars (CD 201, FT 9 and CD 205 in 2004/2005, and CD 219 RR, CD 205 and CD 214 RR in 2005/2006) had similar plastochron values. Considering all cultivars, the average and variation (lowest and greatest) value of plastochron were 55.9 $\left(49.6\right.$ - 60.3) ${ }^{\circ} \mathrm{C}$ day node ${ }^{-1}$ in 2004/2005 (a dry year), and 50.7 (48.1 - 53.9) ${ }^{\circ} \mathrm{C}$ day node ${ }^{-1}$ in 2005/2006 (a wet year), respectively. These results and the fact that the plastochron of the CD 205 cultivar, used in both years, was also greater in the driest year indicate environmental (soil water deficit) effect on the plastochron.

Key words: node appearance, temperature, plant development, degree days, water deficit.

( $\left.{ }^{1}\right)$ Recebido para publicação em 13 de julho de 2006 e aceito em 3 de agosto de 2007.

$\left(^{2}\right)$ Departamento de Fitotecnia, Centro de Ciências Rurais (CCR), Universidade Federal de Santa Maria (UFSM), Av. Roraima, 1000, 97105-900 Santa Maria (RS). E-mail: nstreck1@smail.ufsm.br (*) Autor para correspondência. Bolsista de Produtividade em Pesquisa do CNPq.

$\left({ }^{3}\right)$ Curso de Graduação em Agronomia, CCR, UFSM, Santa Maria (RS), Brasil.

$\left({ }^{4}\right)$ Programa de Pós-graduação em Agronomia, UFSM. Bolsista de Mestrado da Fundação Coordenação de Aperfeiçoamento de Pessoal de Nível Superior (CAPES). 


\section{INTRODUÇÃO}

A soja (Glycine max (L.) Merrill) é a principal fonte de óleo vegetal do mundo. Os principais produtores mundiais são os Estados Unidos da América, Brasil, Argentina e China, responsáveis por mais de $90 \%$ da produção mundial (COSTA, 1996; UsDA, 2005). Na safra 2004/2005, a área cultivada com soja no mundo foi de 92 milhões de hectares com uma produção de aproximadamente 213 milhões de toneladas (UsDa, 2005). O Brasil é o segundo maior produtor mundial de soja e na safra 2004/ 2005 foram cultivados em torno de 22 milhões de hectares, totalizando uma produção cerca de 51 milhões de toneladas do grão (USDA, 2005). A Região Sul do Brasil é a que tem a maior área cultivada, mas sua produção é menor quando comparada com os Estados da Região Centro-Oeste. O Rio Grande do Sul é o segundo maior produtor da Região Sul, onde foram produzidos 9,6 milhões de toneladas do grão na safra 2002/2003 e em 2004/2005, devido à forte estiagem, foram produzidos 2,3 milhões de toneladas do grão (CONAB, 2005). Em função do tamanho da cadeia produtiva do agronegócio da soja, pesquisas com esta cultura têm o potencial de promover importantes ganhos socioeconômicos.

O cálculo da taxa (ou velocidade) de aparecimento de nós na haste principal é um importante componente de modelos de simulação do crescimento, desenvolvimento e rendimento de soja (JONES e LAING, 1978; SinCLAIR, 1986; SinCLAIR et al., 2005). Integrando-se a taxa de aparecimento de nós, tem-se o número total ou acumulado de nós $(\mathrm{NN})$ na haste em um determinado momento do ciclo de desenvolvimento da planta de soja, o que é uma excelente medida de desenvolvimento vegetal. O NN também é usado como indicador dos estádios de desenvolvimento durante o subperíodo vegetativo da soja, como por exemplo, VC (quando o par de folhas unifolioladas está desenrolado) e V1 (quando o primeiro par de folhas unifolioladas está expandido e a primeira folha trifoliada está desenrolada) (JoHNSON, 1997). Outros estádios de desenvolvimento, como o início da floração na soja, também estão associados com o NN (JohNson, 1997). O NN está associado à evolução da área foliar da planta, pois de cada nó surge uma folha trifoliolada com exceção do primeiro nó onde surgem duas folhas unifolioladas. A evolução da área foliar, por sua vez, determina a interceptação da radiação solar, fotossíntese, produção de biomassa e rendimento de grãos (SiNCLAIR, 1986).

A emissão de nós na haste principal pode ser estimada a partir do conhecimento do tempo necessário para o aparecimento de dois nós sucessivos na planta. $\mathrm{O}$ intervalo de tempo entre o aparecimento de nós sucessivos na haste, em dicotiledôneas, é denominado plastocrono (Sinclair, 1984; BAKER e REDDY, 2001; STRECK et al., 2005).

Uma unidade de tempo, bastante utilizada para representar tempo em plantas é a soma térmica, com unidade graus-dia $\left({ }^{\circ} \mathrm{C}\right.$ dia). A soma térmica é a melhor medida de tempo biológico em plantas do que dias do calendário civil, ou seja, dia do ano ou dias após a semeadura ou transplante, pois considera o efeito da temperatura sobre o desenvolvimento vegetal, a qual é um dos principais fatores meteorológicos que governam o desenvolvimento de plantas, incluindo o aparecimento de nós e folhas (GILMORE e ROGERS, 1958; Russele et al., 1984; McMaster e SMika, 1988). O plastocrono, neste caso, é a soma térmica necessária para o aparecimento de um nó na haste da planta e tem como unidade ${ }^{\circ} \mathrm{C}$ dia nó ${ }^{-1}$. Além da temperatura, o déficit hídrico também pode afetar o desenvolvimento da soja, tendo efeito de retardamento do desenvolvimento até a floração e de aceleração do desenvolvimento durante o enchimento das vagens (WoLF, 2002).

O conceito do plastocrono, com unidade de tempo em ${ }^{\circ} \mathrm{C}$ dia, tem sido amplamente empregado para calcular o NN em modelos de simulação do desenvolvimento de espécies dicotiledôneas como a soja (SINCLAIR, 1986), o meloeiro (BAKER et al., 2001; StRECK et al., 2005) e plantas daninhas (DeEn et al., 2001; Weaver, 2003). Freqüentemente, o valor do plastocrono, no entanto, varia com a espécie e com a cultivar dentro da espécie (BAKER et al., 2001; WEAVER, 2003; SincLAir et al., 2005). No Brasil existem várias empresas públicas e privadas que lançam freqüentemente no mercado novas cultivares de soja adaptadas a diferentes regiões edafoclimáticas. Conseqüentemente, existem cultivares de soja disponíveis no mercado nas quais não se conhece o valor do plastocrono, o que constituiu o incentivo para este esforço científico.

O objetivo deste trabalho foi estimar o plastocrono em algumas cultivares de soja, recomendadas para cultivo no Sul do Brasil.

\section{MATERIAL E MÉTODOS}

Foram desenvolvidos dois experimentos em campo na área experimental do Departamento de Fitotecnia da Universidade Federal de Santa Maria, Santa Maria, RS ( $29^{\circ} 43^{\prime} \mathrm{S}, 53^{\circ} 43^{\prime} \mathrm{W}, 95 \mathrm{~m}$ de altitude), sendo um no ano agrícola de 2004/2005 e outro no ano agrícola de 2005/2006. O clima da região, segundo a classificação de Köppen, é Cfa subtropical 
úmido sem estação seca definida com verões quentes (Moreno, 1961). O solo do local é uma transição entre a unidade de Mapeamento São Pedro, um Argissolo Vermelho distrófico arênico e a Unidade de Mapeamento Santa Maria, um Alissolo Hipocrônico argilúvico típico (EMBRAPA, 1999). Em 2004/ 2005 foram utilizadas seis cultivares e em 2005/2006 foram cinco cultivares de soja (Tabela 1). Essas cultivares foram selecionadas por representarem genótipos de ciclos de desenvolvimento distintos e lançadas em diferentes anos e por empresas públicas e privadas diversas (Tabela 1).

Tabela 1. Ciclo, regiões de recomendação, origem e ano de lançamento das cultivares de soja usadas no estudo

\begin{tabular}{lccc}
\hline Cultivar & Ciclo & Recomendação & Origem \\
\cline { 2 - 4 } CD 201 & Sno Agrícola 2004/2005 & Ano* \\
CD 203 & Semiprecoce & RS, SC & Coodetec \\
CD 205 & Semiprecoce & RS, SC & Ocepar /Coodetec \\
FT 9 & Semitardio & RS, SC & Ocepar /Coodetec \\
BRS 66 & Semiprecoce & PR & FT - Pesquisa e sementes \\
FUNDACEP 39 & Médio & RS & Embrapa Trigo / Embrapa Soja \\
& Médio & RS & FUNDACEP \\
CD 205 & & Ano Agrícola 2005/2006 & 1997 \\
CD 209 & Semitardio & RS, SC & Ocepar /Coodetec \\
CD 213 RR & Médio & RS, SC & Coodetec \\
CD 214 RR & Precoce & - & Coodetec \\
CD 219 RR & Precoce & - & Coodetec \\
\hline
\end{tabular}

*Ano de lançamento, - = dado não informado. Coodetec = Cooperativa Central de Pesquisa Agrícola. Ocepar = Organização das Cooperativas do Estado do Paraná. Embrapa = Empresa Brasileira de Pesquisa Agropecuária. FUNDACEP = Fundação Centro de Experimentação e Pesquisa. Fonte: Apassul, 2006; Coodetec, 2006; Fundacep, 2006.

O delineamento experimental foi em blocos ao acaso. Em 2004/2005, utilizaram-se duas parcelas por cultivar e cada parcela (unidade experimental) era formada por três linhas de $3 \mathrm{~m}$ de comprimento e espaçamento de 0,5 m entre linhas. Em 2005/2006, consideraram-se três parcelas e cada parcela era formada por quatro linhas de $4 \mathrm{~m}$ de comprimento e espaçamento de $0,5 \mathrm{~m}$ entre linhas. A densidade de plantas nos dois anos foi de 40 plantas $\mathrm{m}^{-2}$. A adubação seguiu as recomendações para a cultura da soja (RPSRS, 2000) e outros tratos culturais, como o controle de plantas daninhas, foram feitos de maneira que as plantas se mantivessem livres de interferência de fatores bióticos.

As semeaduras foram realizadas em 3/12/ 2004 e $23 / 11 / 2005$, respectivamente, nos dois anos agrícolas. A emergência das plântulas foi determinada em cada parcela contando-se diariamente o número de plantas visíveis acima do nível do solo. Com esses dados, calculou-se a data de $50 \%$ das plantas emergidas em cada parcela. Uma semana após a emergência, em cada parcela, foram identificadas cinco plantas na linha central, com arames coloridos, no ano agrícola de 2004/2005 e seis plantas nas duas linhas centrais no ano agrícola de 2005/2006. Nas plantas marcadas, contou-se o número de nós visíveis na haste principal três vezes por semana. Um nó foi considerado visível quando a folha associada a ele tinha as bordas de pelo menos um limbo foliar desenrolado não mais se tocando (JoHNSON, 1997). Cada planta marcada foi considerada uma amostra da parcela e a média das plantas marcadas em cada parcela foi considerada uma repetição, método usado por STRECK et al. (2005).

Os dados de temperatura mínima e máxima diária do ar e precipitação pluvial durante o período experimental foram registrados na Estação Climatológica Principal do $8 .^{\circ}$ Distrito de Meteorologia do Instituto Nacional de Meteorologia, localizada aproximadamente a $300 \mathrm{~m}$ da área experimental.

A soma térmica diária $\left(\mathrm{STd},{ }^{\circ} \mathrm{C}\right.$ dia), a partir da emergência, foi calculada, de acordo com GiLmore e Rogers (1958) e ARNOLD (1960):

$$
\mathrm{STd}=(\mathrm{Tmed}-\mathrm{Tb}) \cdot 1 \text { dia } \quad(\text { eq. } 1)
$$


em que: Tmed é a temperatura média do ar, calculada pela média aritmética entre as temperaturas mínima e máxima diárias do ar e Tb é a temperatura base para emissão de nós da soja, assumida $10{ }^{\circ} \mathrm{C}$ (Brown,1960; Major et al., 1975; Piper et al., 1996). Considerou-se que se Tmed $<$ Tb então Tmed $=\mathrm{Tb}$ e, se Tmed $>30{ }^{\circ} \mathrm{C}$, então Tmed $=30{ }^{\circ} \mathrm{C}$ (Brown, 1960; MAJOr et al., 1975; PIPER et al., 1996).

A soma térmica acumulada $\left(\mathrm{STa},{ }^{\circ} \mathrm{C}\right.$ dia), a partir do dia de emergência, foi calculada por:

$$
\mathrm{STa}=\Sigma \mathrm{STd} \quad \text { (eq. } 2)
$$

O balanço hídrico climatológico foi calculado desde a emergência das plantas pelo método de Thornthwaite e Matter (1955), utilizando-se valores diários de temperatura média, precipitação pluvial e evapotranspiração potencial estimada pelo método de Penman, e considerando uma capacidade de armazenamento de água no solo de 104 mm (Peraza, 2003).

Para cada repetição (média de cinco plantas em 2004/ 2005 e média de seis plantas em 2005/2006) obteve-se a regressão linear entre o número de nós acumulados (NN) na haste principal e a soma térmica acumulada (STa) a partir da emergência (SINCLAIR, 1984; BAKER e REDDY, 2001; STRECK et al., 2005). Na regressão linear utilizou-se o NN até 20 nós. O plastocrono $\left({ }^{\circ} \mathrm{C}\right.$ dia nó $\left.{ }^{-1}\right)$ foi considerado como o inverso do coeficiente angular da regressão linear entre NN e STa (KLEPPER et al., 1982; KIRBY, 1995; BAKER e REDDY, 2001). Obteve-se um valor de plastocrono para cada repetição (STRECK et al., 2005) e as médias das cultivares em cada ano foram comparadas pelo teste de Duncan a $5 \%$ de probabilidade.

\section{RESULTADOS E DISCUSSÃO}

As condições meteorológicas, no que se refere à temperatura do ar e precipitação pluvial, durante o período de emissão de folhas da soja são apresentadas na Figura 1. A média das temperaturas médias diárias do ar durante a emissão de folhas foi $25,1{ }^{\circ} \mathrm{C}$ e 25,2 ${ }^{\circ} \mathrm{C}$ em 2004/ 2005 e 2005/ 2006 respectivamente (Figura 1a). Apenas dois dias (2/2 e 3/2/2005) em 2004/2005 foram marcadamente de temperaturas mais baixas $\left(15,6\right.$ e $\left.18,4^{\circ} \mathrm{C}\right)$, mas ainda assim acima da $\mathrm{Tb}$. Em geral, as temperaturas médias diárias do ar estiveram quase sempre abaixo da temperatura ótima $\left(30^{\circ} \mathrm{C}\right)$, com exceção de três dias em janeiro de 2005 e quatro dias em janeiro de 2006 (Figura 1b). O ano agrícola de 2004/2005 foi mais seco do que o ano agrícola $2005 / 2006$, com um total acumulado de chuva durante o período de emissão de folhas de $142,4 \mathrm{~mm}$ e 302,8 $\mathrm{mm}$ respectivamente. Não foi realizada nenhuma irrigação durante o período de emissão de folhas e não foram verificados sintomas visíveis de deficiência hídrica nas plantas de soja nos dois anos do estudo, mas os valores de déficit hídrico calculados no balanço hídrico climatológico alcançaram valores de $30,3 \mathrm{~mm}$, $39,1 \mathrm{~mm}$ e $44,5 \mathrm{~mm}$ em três ocasiões no ano agrícola $2004 / 2005$ e $25,9 \mathrm{~mm}$ e $24 \mathrm{~mm}$ em duas ocasiões no ano agrícola 2005/2006 (Figura 1c).
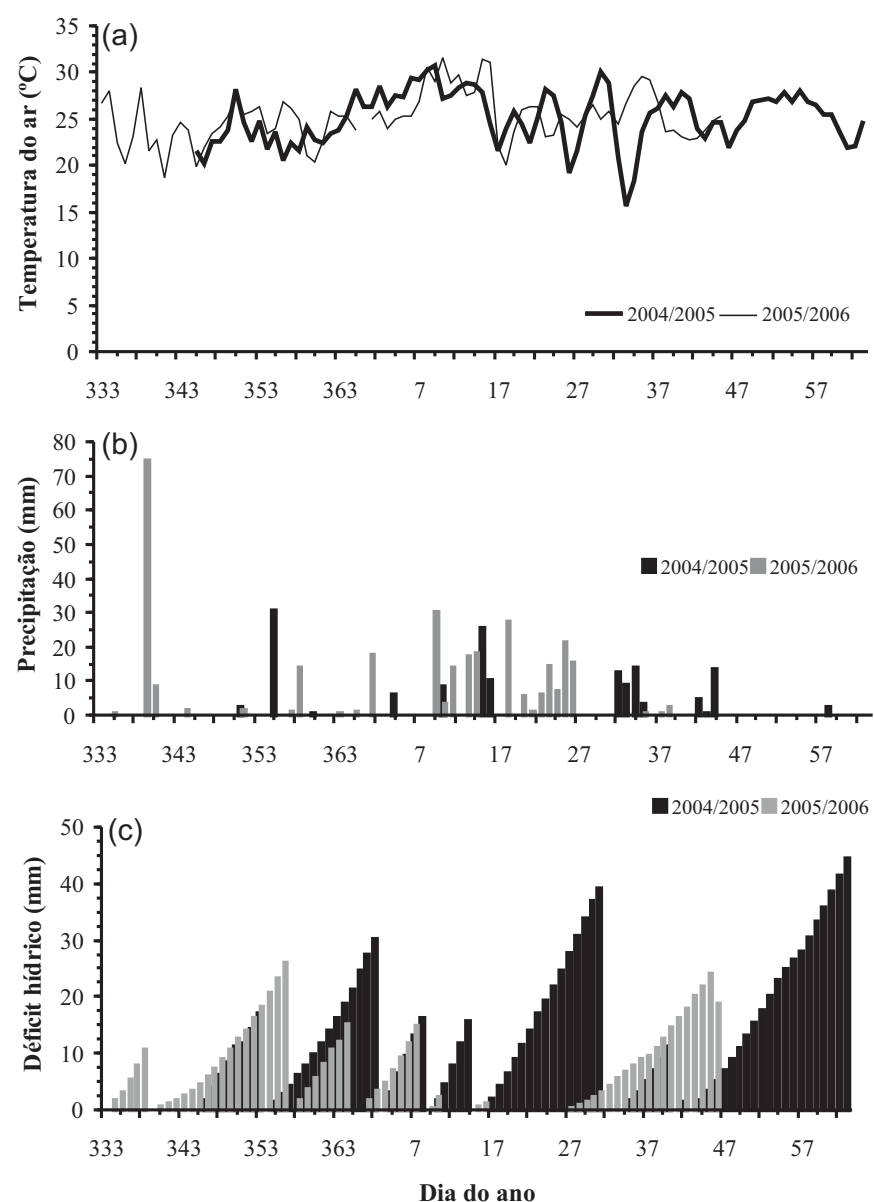

Figura 1. Temperatura média diária do ar (a), precipitação pluvial diária (b) e déficit hídrico diário no solo (c) durante o período de emissão de nós da soja nos anos agrícolas de 2004/ 2005 e 2005/ 2006 em Santa Maria (RS).

Valores elevados de correlação entre NN e STa $\left(R^{2}>0,99\right)$ foram verificados para todas as repetições (média das plantas marcadas em cada parcela) das cultivares nos dois anos agrícolas. Dois exemplos da relação entre número de nós acumulados na haste principal (NN) e a soma térmica acumulada (STa), para duas cultivares (CD 203 e FUNDACEP 39) no ano agrícola 2004/2005, são apresentados na Figura 2. A elevada linearidade entre NN e STa indica a temperatura do ar como um importante fator ambiental que governa o aparecimento de nós em soja e a estimativa do plastocrono pelo método da regressão linear é um método apropriado (SINCLAIR et al., 2005; STRECK et al., 2005). 

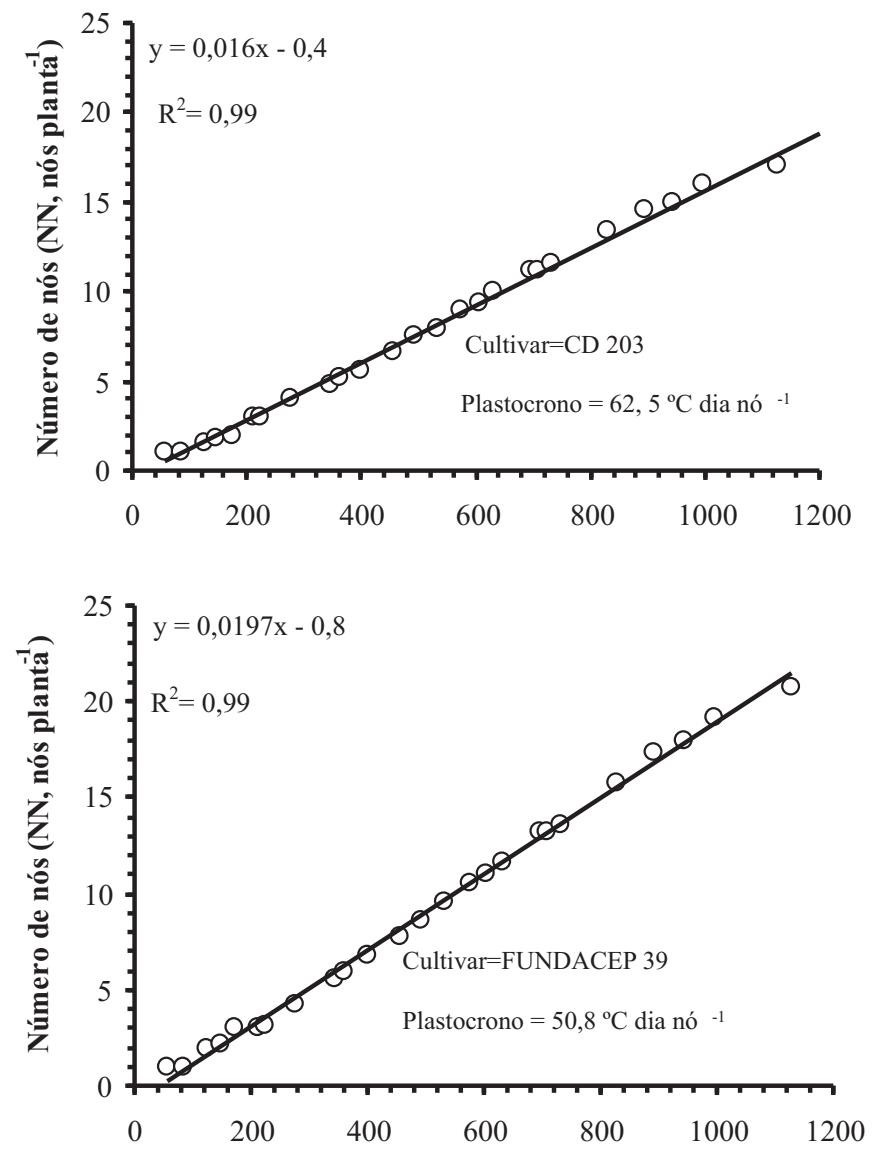

\section{Soma térmica acumulada $\left(\mathrm{STa},{ }^{0} \mathrm{C}\right.$ dia $)$}

Figura 2. Relação entre número de nós acumulados na haste principal (NN, nós planta ${ }^{-1}$ ) e soma térmica acumulada (STa, ${ }^{\circ} \mathrm{C}$ dia), utilizada na estimativa do plastocrono em uma repetição (parcela com cinco plantas observadas) de duas cultivares de soja. Cada ponto representa a média de cinco plantas. Santa Maria (RS), 2004/2005.

O plastocrono variou de 49,6 a $60,3{ }^{\circ} \mathrm{C}$ dia nó ${ }^{-1}$ entre as cultivares em $2004 / 2005$ e de 48,1 a $53,9^{\circ} \mathrm{C}$ dia nó ${ }^{-1}$ em 2005/2006 (Tabela 2). Essa variação de plastocrono é similar à variação de 47,7 a $64,2{ }^{\circ} \mathrm{C}$ dia nó $^{-1}$ entre oito cultivares de soja observada por SINCLAIR et al. (2005). Em 2004/2005, nas cultivares CD 203 e BRS 66, verificaram-se os maiores valores de plastocrono, diferindo da FUNDACEP 39. No entanto, essas três cultivares não diferiram da CD 201, CD 205 e FT 9, que tiveram valores intermediários de plastocrono (Tabela 2). Em 2005/2006, a cultivar CD 209 com o maior valor de plastocrono, diferiu apenas da 'CD 213 RR'. Essas duas cultivares não diferiram da CD 219 RR, CD 205 e CD 214 RR (Tabela 2). Diferenças de plastocrono entre cultivares da mesma espécie também foram reportadas em meloeiro por BAKER e REDDY (2001) e em soja por SINCLAIR et al. (2005).
As diferenças de plastocrono entre cultivares não estiveram relacionadas ao ciclo da cultivar. Por exemplo, em 2004/2005, em um mesmo agrupamento de cultivares pelo teste de Duncan (agrupados por " $a$ " na Tabela 2), há cultivares representantes desde o grupo semiprecoce (CD 203, BRS 66, FT 9) até o tardio (CD 205). Verificou-se a mesma tendência em 2005/ 2006, quando as cultivares foram da mesma empresa (Coodetec), com uma cultivar precoce (CD 214 RR) tendo plastocrono similar a uma cultivar tardia (CD 205). Esses resultados concordam com aqueles relatados por SINCLAIR et al. (2005), que também não observaram relação entre velocidade de emissão de nós e ciclo de desenvolvimento de oito cultivares de soja, e confirmam resultados anteriores que o ciclo de desenvolvimento da soja não é associado com a velocidade com que os nós aparecem, mas sim com o número final de nós (CRISWELl e HuME,1972).

Tabela 2. Valores do plastocrono em cultivares de soja cultivadas na região de Santa Maria, RS, nos anos agrícola de 2004/2005 e 2005/2006

\begin{tabular}{|c|c|}
\hline Cultivar & Plastocrono $\left({ }^{\circ} \mathrm{C}\right.$ dia nó $\left.{ }^{-1}\right)$ \\
\hline & Ano Agrícola 2004/2005 \\
\hline CD 203 & $60,3 \mathrm{a}$ \\
\hline BRS 66 & 59,8 a \\
\hline CD 201 & $56,5 \mathrm{ab}$ \\
\hline FT 9 & $54,5 \mathrm{ab}$ \\
\hline CD 205 & $54,4 \mathrm{ab}$ \\
\hline FUNDACEP 39 & $49,6 \mathrm{~b}$ \\
\hline Média e Desvio-padrão & $55,9( \pm 4,0)$ \\
\hline
\end{tabular}

$\begin{array}{lc} & \text { Ano Agrícola 2005 } \\ \text { CD 209 } & 53,9 \mathrm{a} \\ \text { CD 219 RR } & 50,8 \mathrm{ab} \\ \text { CD 205 } & 50,4 \mathrm{ab} \\ \text { CD 214 RR } & 50,2 \mathrm{ab} \\ \text { CD 213 RR } & 48,1 \mathrm{~b}\end{array}$

Média e Desvio-padrão $50,7( \pm 2,0)$

Médias seguidas pela mesma letra minúscula na vertical em cada ano agrícola não diferem entre si pelo teste de Duncan a 5\% de probabilidade de erro.

A cultivar CD 205 foi avaliada nos dois anos, com valor de plastocrono maior em 2004/2005 (54,4 ${ }^{\circ} \mathrm{C}$ dia nó $\left.{ }^{-1}\right)$ do que em 2005/2006 (50,4 ${ }^{\circ} \mathrm{C}$ dia nó $\left.{ }^{-1}\right)$. Esta diferença de $4,0{ }^{\circ} \mathrm{C}$ dia nó ${ }^{-1}$ no plastocrono da cultivar CD 205 nos dois anos foi significativa ( $p<$ $0,05)$. Uma hipótese que pode ser levantada para explicar a diferença de plastocrono da cultivar CD 205 em anos agrícolas distintos é a diferença de déficit hídrico, sendo o ano de 2004/2005 mais seco do que 2005/2006 (Figura 1c). 
Quando ocorre deficiência hídrica no solo, a temperatura da folha de soja pode ser de 5 a $10{ }^{\circ} \mathrm{C}$ maior do que em plantas sem deficiência hídrica (JuNG e ScotT, 1980; Cox e Jolliff, 1987) devido à redução na transpiração em vista do fechamento estomático (Tuner e BegG, 1981; Hashimoto et al., 1984; Hatfield et al., 1987). Como no ano agrícola de 2004/2005 as deficiências hídricas no solo foram maiores (Figura 1c), é possível que, embora não fosse notado nenhum sintoma aparente nas folhas, o fechamento estomático em nível diário tenha sido por mais tempo, e com isso a temperatura da folha tenha sido maior que a temperatura ótima $\left(30^{\circ} \mathrm{C}\right)$ nesse ano. Essa hipótese poderia explicar o retardamento do desenvolvimento (maior plastocrono) no ano de 2004/2005, concordando com a afirmação de Wolf (2002) de que o déficit hídrico no solo retarda o desenvolvimento vegetativo da soja.

O valor médio de plastocrono obtido no primeiro ano $\left(55,9^{\circ} \mathrm{C}\right.$ dia nó $\left.{ }^{-1}\right)$ e no segundo ano $(50,7$ ${ }^{\circ} \mathrm{C}$ dia nó ${ }^{-1}$ ) é similar e menor, respectivamente, ao valor de $55,5{ }^{\circ} \mathrm{C}$ dia nó ${ }^{-1}$ obtido por Sinclair (1986) para simular o aparecimento de folhas em seu modelo de soja. Dentre as cultivares avaliadas, várias tiveram valores similares, como por exemplo, CD 201, FT 9 e CD 205 no ano de 2004/2005 (em torno de $55{ }^{\circ} \mathrm{C}$ dia nó $^{-1}$ ) e CD 219 RR, CD 205 e CD 214 RR em 2005/ 2006 (em torno de $50{ }^{\circ} \mathrm{C}$ dia nó ${ }^{-1}$ ). Sinclair et al. (2005) observaram diferenças estatísticas entre oito cultivares de soja, mas na maioria (seis cultivares), o plastocrono foi aproximadamente igual a $50{ }^{\circ} \mathrm{C}$ dia nó $^{-1}$. A implicação desta similaridade de plastocrono em várias cultivares de soja é que, em modelos de simulação do aparecimento de nós, um valor único de plastocrono pode representar várias cultivares, $\mathrm{o}$ que torna o modelo mais robusto e de fácil aplicabilidade. Por outro lado, o fato de que algumas cultivares tiveram plastocrono diferentes há uma implicação importante, quando o conceito do plastocrono é usado para simular o aparecimento de folhas ou nós, como no modelo de SincLaIR (1986). Por exemplo, assumindo que uma cultivar produza 20 nós na haste principal (Figura 2), a diferença de $10,7^{\circ} \mathrm{C}$ dia nó ${ }^{-1}$ entre o maior e o menor plastocrono observado no experimento em $2004 / 2005$ e de $5,5{ }^{\circ} \mathrm{C}$ dia nó ${ }^{-1}$ em $2005 / 2006$ resulta em $214{ }^{\circ} \mathrm{C}$ dia e 110 ${ }^{\circ} \mathrm{C}$ dia, respectivamente, para emissão do último nó. Esse processo resultará em vários dias do calendário civil, especialmente se durante a fase de emissão de nós ocorrer temperaturas amenas abaixo da temperatura ótima $\left(<30{ }^{\circ} \mathrm{C}\right)$. Essas diferenças de plastocrono em algumas cultivares também podem levar a erros na estimativa da área foliar, quando sua evolução é calculada em função do aparecimento de nós, e na estimativa da data de estádios de desenvolvimento (JOHNSON, 1997).

\section{CONCLUSÕES}

1. As cultivares (CD 201, FT 9 e CD 205 em 2004/2005 e CD 219 RR, CD 205 e CD 214 RR em $2005 / 2006)$ obtiveram valores similares de plastocrono (em torno de 55 e $50{ }^{\circ} \mathrm{C}$ dia nó ${ }^{-1} \mathrm{em} 2004$ / 2005 e 2005/2006 respectivamente); em outras cultivares (CD 203, BRS 66 e FUNDACEP 39 em 2004/ 2005, e CD 209 e CD 213 RR, em 2005/2006) houve diferenças de plastocrono, o que deve ser levado em conta em modelos de simulação de crescimento, desenvolvimento e rendimento desta espécie, que usam o conceito do plastocrono.

2. O tempo médio necessário para o aparecimento de dois nós sucessivos em soja, considerando todas as cultivares, foi maior no ano mais seco do que no mais úmido, tanto considerando todas as cultivares juntas $\left(55,9^{\circ} \mathrm{C}\right.$ dia nó ${ }^{-1}$ no ano mais seco e $50,7^{\circ} \mathrm{C}$ dia nó ${ }^{-1}$ no ano mais úmido) como considerando a cultivar CD 205 usada nos dois anos $\left(54,4{ }^{\circ} \mathrm{C}\right.$ dia nó ${ }^{-1}$ no ano mais seco e $50,4{ }^{\circ} \mathrm{C}$ dia nó ${ }^{-1}$ no ano mais úmido), o que indica efeito do ambiente (déficit hídrico no solo) sobre o plastocrono.

\section{AGRADECIMENTOS}

Os autores agradecem aos revisores Ad Hoc pelas valiosas sugestões em versões anteriores do manuscrito. Aos estudantes de Agronomia da UFSM Lidiane Cristine Walter e Hamilton Telles Rosa pelo auxílio na coleta dos dados durante o experimento do primeiro ano.

\section{REFERÊNCIAS}

ARNOLD, C.Y. Maximum-minimum temperatures as a basis for computing heat units. Proceedings of the American Society for Horticultural Sciences, Alexandria, v.76, n.1, p.682-692, 1960.

APASSUL. Associação dos Produtores e Comerciantes de Sementes do Rio Grande do Sul. Cultivares/Descrição. Disponível em : Http://www.apassul.com.br/ conteudo.asp? content $=12 \& a=$ view $\& I D=12$. Acesso em 28 mar. 2006.

BAKER, J.T.; REDDY, V. R. Temperature effects on phenological development and yield of muskmelon. Annals of Botany, Oxford, v.87, p.605-613, 2001.

BAKER, J.T.; LESKOVAR, D.I.; REDDY, V.R.; DAINELLOS, F.J. A simple phenological model for muskmelon development. Annals of Botany, Oxford, v.87, p.615-621, 2001.

BROWN, D.V. Soybean ecology: I. Development-temperature relationships from controlled environment studies. Agronomy Journal, Madison, v.52, n.9, p.493-496, 1960. 
COODETEC. Cooperativa Central de Pesquisa Agrícola. Produtos/ Soja. Disponível em: http://www2.coodetec.com.br/coodetec/ listProdutos.action?culturaId=3. Acesso em 28 mar. 2006.

CONAB. Série histórica de área e produção plantada por unidades da federação. Disponível na internet: http:// www.conab.gov.br/download/safra/sojaseriehist.xls. Acesso em 20 dez. 2005.

COSTA, J. A. Cultura da soja. Porto Alegre: Evangraf, 1996. 233p.

COX, W.J.; JOLLIFF, G.D. Crop-water relations of sunflower and soybean under irrigated and dry land conditions. Crop Science, Madison, v.27, p.553-557, 1987.

CRISWELL, J. G.; HUME, D. J. Variation in sensitivity to photoperiod among early maturing soybean strains. Crop Science, Madison, v.12, p. 657-660, 1972.

DEEN, W.;SWANTON, C.J.; HUNT, L.A. A mechanistic growth and development model of common ragweed. Weed Science, Champaign, v. 49, p.723-731, 2001.

EMBRAPA. Centro Nacional de Pesquisa de Solos (Rio de Janeiro, RJ). Sistema Brasileiro de Classificação de solos. Brasília: EMBRAPA - SPI e EMBRAPA - CNPS, 1999. 412p.

FUNDACEP. Fundação Centro de Experimentação e Pesquisa. Sementes/ Soja. Disponível em: http:// www.fundacep.com.br. Acesso em 28 mar. 2006.

GILMORE, E.C. JR.; ROGERS, J.S. Heat units as a method of measuring maturity in corn. Agronomy Journal, Madison, v.50, n.10, p.611-615, 1958.

HASHIMOTO, Y.; INO T.; KRAMER, P.J.; NAYLOR, A.W.; STRAIN, B.R. Dynamic analysis of water stress of sunflower leaves by means of a thermal image processing system. Plant Physiology, v.76, p.266-269, 1984.

HATFIELD, J.L.; QUISENBERRY, J.E.; DILBECK, R.E. Use of canopy temperatures to identify water conservation in cotton germoplasm. Crop Science, Madison, v.27, p.269-273, 1987.

JOHNSON, S.R. How a soybean plant develops. Ames: Iowa Stale University of Science and Technology, and Cooperative Extension Service, 1997. 18 p. (Special Report n.53)

JONES, P. G.; LAING, D. R. Simulation of the phenology of soybean. Agricultural Systems, v. 3, n. 4, p. 295-311, 1978.

JUNG, P.K.; SCOTT, H.D. Leaf water potential, stomatal resistance and temperature relations in field-grown soybeans. Agronomy Journal, Madison, v.72, p.986-990, 1980.

KLEPPER, B.; RICKMAN, R.W.; PETERSON, C.W. Quantitative characterization of vegetative development in small cereal grains. Agronomy Journal, Madison, v.74, p.780-792, 1982.

KIRBY, E.J. Factors affecting rate of leaf emergence in barley and wheat. Crop Science, Madison, v.35, n.1, p.11-19, 1995.

MAJOR, D. J.; JOHNSON, D.R.; TANNER, J.W.; ANDERSON, I.C. Effects of daylength and temperature on soybean development. Crop Science, Madison, v. 15, p. 174-179, 1975.
MCMASTER, G.S.; SMIKA, D.E. Estimation and evaluation of winter wheat phenology in the central Great Plains. Agricultural and Forest Meteorology, Amsterdam, v.43, n.1, p.1-18, 1988.

MORENO, J. A. Clima do Rio Grande do Sul. Porto Alegre: Secretaria da Agricultura, 1961. 46p.

PERAZA, J.E.S. Retenção de água e pedofunções para solos do Rio Grande do Sul. 2003. 117 f. Dissertação (Mestrado Engenharia Agrícola) - Universidade Federal de Santa Maria, Santa Maria, 2003.

PIPER, E.; BOOTE, K.J.; JONES, J.W.; GRIMM, S.S. Comparison of two phenology models for predicting flowering and maturity date of soybean. Crop Science, Madison, v.36 p.16061614, 1996.

RPSRS [Reunião de Pesquisa de soja da Região Sul]. Recomendações técnicas para a cultura da soja no Rio Grande do Sul e Santa Catarina 2000/2001. Santa Maria: UFSM/CCR/ Departamento de Defesa Fitossanitária, 2000. 148p.

RUSSELE, M.P.; WILHELM, W.W.; OLSON, R.A.; POWER, J.F. Growth analysis based on degree days. Crop Science, Madison, v.24, n.1, p.28-32, 1984.

SINCLAIR, T.R. Leaf area development in field grown soybean. Agronomy Journal, Madison, v.76, n.1, p.141-146, 1984.

SINCLAIR, T.R. Water and nitrogen limitations in soybean grain productivity. I. Model development. Field Crops Research, Amsterdam v.15, n.2, p.125-141, 1986.

SINCLAIR, T.R.; NEUMAIER, N.; FARIAS, J.R.B.; NEPOMUCENO, A.L. Comparison of vegetative development in soybean cultivars for low-latitude environment. Field Crops Research, Amsterdam, v. 92, n. 1, p.53-59, 2005.

STRECK, N.A.; TIBOLA, T.; LAGO, I.; BURIOL, G.A.; HELDWEIN, A.B.; SCHNEIDER, F. M.; ZAGO, V. Estimativa do plastocrono em meloeiro (Cucumis melo L.) cultivado em estufa plástica em diferentes épocas do ano. Ciência Rural, Santa Maria, v.35, n.6, p.1275-1280, 2005.

THORNTHWAITE, C.W.; MATHER, J.R. The water balance. Centerton: Institute of Tecnology, Laboratory of Climatology, 1955. 104p.

TUNNER, N.C.; BEGG, J.E. Plant-water relations and adaptation to stress. Plant and Soil, Dordrecht, v.58, p.97-131, 1981.

USDA. Soybean area, yield and production: Capturado em 20 dez. 2005. Online. Disponível na Internet http:// www.faz.usda.gov/psd.

WEAVER, S.E. Correlations among relative crop and weed growth stages. Weed Science, Champaign, v.51, p.163-170, 2003.

WOLF, J. Comparison of two soybean simulation models under climate change. I. Model calibration and sensitivity analyses. Climate Research, Silver Spring, v.20, p.55-70, 2002 